\title{
El sistema de riego parcelario como herramienta para el desarrollo de comunidades rurales. Evaluación del caso cantón Mocache- provincia de Los Ríos-Ecuador
}

\author{
URL: http://revistas.uta.edu.ec/erevista/index.php/bcoyu/article/view/692ＤOl: http://dx.doi.org/10.31164/bcoyu.21.2019.692
}

\section{León Argüello-Núñez'; Washington Argüello-Núñez²; Mario Pérez-Arévalo ${ }^{3}$}

Fecha de recepción: 31 de enero de 2019

Fecha de aceptación: 3 de junio de 2019

\section{Resumen}

El objetivo de este estudio es evaluar el proyecto de riego "Mejoramiento de la producción agrícola a través de la implementación de sistemas agroforestales y riego parcelario, Cantón Mocache-Provincia de Los Ríos", ejecutado por el Gobierno Autónomo Descentralizado Provincial de Los Ríos para favorecer el desarrollo productivo del sector. Este trabajo evalúa los resultados, el impacto socioeconómico y ambiental generado por la puesta en marcha del proyecto mencionado. Para obtener los resultados se encuestaron a las familias del sector. Entre los principales resultados tenemos que los agricultores sí se han beneficiado con este proyecto, por cuanto han podido diversificar su producción agrícola, implementado sistemas agroforestales y mejorado sus condiciones de vida.

Palabras clave: Seguridad alimentaria; desarrollo rural; pequeños agricultores; riego parcelario.

\begin{abstract}
The objective of this study is to evaluate the irrigation project "Improvement of agricultural production through the implementation of agroforestry systems and irrigation parcelario, Canton Mocache-Province of Los Ríos", executed to promote the productive development of the sector. This work evaluates the results, the socioeconomic and environmental impact generated by the implementation of the aforementioned project. For which, the families of the sector were surveyed. Among the main results we have that farmers have benefited with this project, because they have been able to diversify their agricultural production, implemented agroforestry systems and improved their living conditions.
\end{abstract}

Keywords: Food safety, rural development, small farmers, parcel irrigation.

\section{Introducción}

El objetivo del desarrollo rural es mejorar las condiciones de vida de las comunidades rurales, con el apoyo y el aprovechamiento de sus propios recursos. Lo que se busca es: un agricultor mejor capacitado, formado, organizado y por otro lado obtener más productividad en sus tierras, incrementar los rendimientos en sus cultivos, pero conservando el entorno y respeto con el medio natural (Martínez de Anguita d' Huart, 2006). Uno de los recursos que tiene mayores beneficios potenciales para los agricultores, porque les permite incrementar la productividad y los ingresos, es el riego (FAO, 2004). De la misma forma, permite elevar los rendimientos de la superficie, introducir nuevos cultivos y aprovechar de manera intensa la tierra, a la vez que beneficiaría al agricultor en vista de que el valor de la tierra se incrementaría (Juárez, 2008).

Los avances tecnológicos que se incorporan a la producción agrícola han permitido la seguridad alimentaria y será muy importante en la sostenibilidad del territorio (Lozano y Restrepo, 2014), asegurando que la población tenga acceso a los alimentos que demanden. En este contexto, es necesario que se dinamice la agricultura a través de un nuevo enfoque de desarrollo agrario en función de la gestión social del riego, buscando la sostenibilidad del territorio, que sea amigable con el ambiente, optimizando los recursos suelo y agua; pero de una manera participativa, buscando alternativas que se adapten a los cambios climáticos extremos, proponiendo suficientes alimentos sanos y nutritivos que vengan de pequeños y medianos agricultores, respetando la biodiversidad, e implementando formas de producciones tradicionales y ancestrales.
Desde el punto de vista que orienta las acciones de los Gobiernos Provinciales a garantizar la soberanía alimentaria en los territorios cabe plantear un cuestionamiento que brinde un norte a las intervenciones en campo:

¿De qué forma pueden las políticas, planes, programas y proyectos, ayudar a las familias a salir de la pobreza y lograr la seguridad alimentaria?

Esta es la pregunta que como Gobiernos Provinciales deben responder en el territorio, haciendo referencia al mandato constitucional establecido en el artículo 263, numerales 5, 6, y 7 de la Constitución de la República del Ecuador (2008), que se centra en el fomento productivo y accesibilidad a servicios de riego, que tenemos como competencia directa de nuestras administraciones.

Bajo este enfoque, los Gobierno Provinciales cumplen un rol sumamente importante en las políticas de consumo de alimentos, desde el punto de partida de la producción primaria en territorio, pues tienen que orientar un trabajo que garantice el fomento a actividades que fortalezcan la soberanía y seguridad alimentaria; desde una visión de fortalecimiento de sistemas de producción diversificados que incorporen en su gestión saberes ancestrales, pero sobre todo accesibilidad al agua.

Sabemos que la zona rural es un motor productivo de la economía popular y solidaria caracterizada por la generación de empleo pero carente de asistencia técnica especializada para la optimización de sus procesos productivos, por lo que es deber de los Gobiernos Provinciales, definir las políticas específicas y diferenciadas que contribuyan a una producción que satisfaga la demanda interna y que promueva el consumo de estos productos con la finalidad de evitar la escasez, ya que se contaría con una oferta suficiente de productos para consumo local e incluso exportables y de alta calidad, aportando a la seguridad alimentaria de la población.

Los gobiernos autónomos descentralizados tienen la obligación de promover el desarrollo de proyectos que brinden acceso a la infraestructura necesaria para la producción, intercambio y comercialización directa entre pequeños productores y consumidores, en mutuo beneficio, como una nueva relación de economía social y solidaria.

Esto permitirá disminuir la migración campo-ciudad, lo que genera a nivel urbano un creciente índice de desempleo y a nivel rural una disminución de la producción primaria, lo que a su vez genera escasez y por ende un aumento del costo de los productos agrícolas en los mercados finales.

El punto estratégico desde los Gobiernos Provinciales con su conocimiento íntegro del territorio y sus capacidades productivas está en definir ¿qué producir?, ¿cómo producir? y ¿para quién producir?, siendo necesario reconocer el potencial de cada territorio y valorar la especialización en su conocimiento por parte del productor, en donde el Gobierno Provincial juega un papel decisivo para fortalecer capacidades, estimular la agricultura asociativa campesina y las actividades de la economía popular y solidaria en el sector rural, brindando la oportunidad de realizar actividades agro productivas y agro económicas a grupos organizados de productores en cada territorio, administrando de esta manera los medios de producción con los cuales trabajan. 
Por todo esto, es necesario considerar como herramienta estratégica para conseguir los objetivos, la elaboración de proyectos, ya que se constituyen en la expresión técnica a las soluciones de los problemas de personas e instituciones. En definitiva, buscar beneficios para la comunidad, en general donde existe pobreza y marginación, posteriormente se hace una evaluación (ex post) a su aplicación para conocer si, una vez concluido el proyecto, ha generado el impacto y resultados esperados (Baca y Herrera, 2016). Un proyecto social de esta naturaleza debe ser económicamente factible, respetuoso con el medio ambiente y que no contamine el entorno.

\section{Breve descripción del proyecto}

El objetivo del proyecto de riego que se evalúa fue contribuir al mejoramiento de la calidad de vida de 60 familias del Recinto Estrella Grande del sector sur del Cantón Mocache, a través de la implementación de un sistema de riego parcelario en el sector como gestión del Gobierno Autónomo Descentralizado Provincial de Los Ríos-GADP y favorecer el desarrollo productivo de este sector. El proyecto se implementó en marzo de 2015 con una duración de 20 meses, en mayo del 2018 se evaluó el proyecto con el objetivo de verificar el cumplimiento de los resultados deseados.

La Prefectura ha logrado una transformación en el tema de riego, drenaje y dragas en la provincia, realizando trabajos de mantenimiento y mejoras en los sistemas de riego, como en el caso de Catarama; ex Comisión de Estudios para el Desarrollo de la Cuenca del Río Guayas-Cedege en Babahoyo y Loma de Coco en Quinsaloma, consiguiendo ayuda técnica de la Agencia de Cooperación Internacional de Japón-JICA, desde que asumió esta competencia transferida por el Gobierno Nacional, para fortalecer el sistema de riego, a fin de aprovechar el agua e incrementar la productividad agrícola en la Provincia de Los Ríos (Gobierno Autónomo Descentralizado Provincial de Los Ríos, 2016).

En el Art. 263 de la Constitución de la República se establece que las competencias de riego son exclusivas de los Gobiernos Provinciales, en la parte pertinente se manifiesta lo siguiente: "Planificar, construir, operar y mantener sistemas de riego, en el ámbito de sus competencias y territorio, y en uso de sus facultades, expedirán ordenanzas provinciales". De la misma forma el Gobierno Nacional impulsa el Plan Nacional de Riego y Drenaje proyectado hasta el 2027. El objetivo principal es ampliar la cobertura y mejorar la eficiencia del riego, mediante el fortalecimiento de las organizaciones de regantes, para que pueda asumir la co-gestión y gestión de los sistemas de riego y drenaje de manera sostenible y eficiente. Este Plan prioriza a sectores sociales que históricamente se han mantenido postergados, es decir, a los pequeños y medianos productores, que son quienes garantizan la soberanía alimentaria del país (Ministerio de Agricultura y Ganadería. Plan Nacional de Riego y Drenaje, 2012).

Este proyecto tiene total relación con el objetivo general del Plan Nacional de Riego y Drenaje, que es la de contribuir al mejoramiento del ingreso de la población y la productividad agropecuaria, en armonía con los principios del buen vivir y la soberanía alimentaria.

Para llevar a cabo este proyecto denominado "Mejoramiento de la producción agrícola a través de la implementación de sistemas agroforestales y riego parcelario, Cantón Mocache, Provincia de Los Ríos", se creó la Asociación de Trabajadores Agrícolas Estrella Grande, domiciliada en el Recinto Estrella Grande del Cantón Mocache, con acuerdo Ministerial No 01605 del 01 de septiembre del 2016. Es una organización de pequeños productores, cuyo objetivo principal es la producción y comercialización de productos agropecuarios. Consta de 76 miembros y poseen sus propios estatutos y reglamentos. Es una organización sin fines de lucro perteneciente a la Economía Popular Solidaria.

De acuerdo a la Ley Orgánica de Economía Popular y Solidaria en la sección 2 sobre las Organizaciones del Sector Asociativo en los artículos 18, 19, la asociación de agricultores Estrella Grande está enmarcada en lo siguiente:

Art. 18.- Sector Asociativo. - Es el conjunto de asociaciones constituidas por personas naturales con actividades económicas productivas similares o complementarias, con el objeto de producir, comercializar y consumir bienes y servicios lícitos y socialmente necesarios, auto abastecerse de materia prima, insumos, herramientas, tecnología, equipos y otros bienes, o comercializar su producción en forma solidaria y auto gestionada bajo los principios de la presente Ley.

Art. 19.- Estructura Interna. - La forma de gobierno y administración de las asociaciones constarán en su estatuto social, que preverá la existencia de un órgano de gobierno, como máxima autoridad; un órgano directivo; un órgano de control interno y un administrador, que tendrá la representación legal; todos ellos elegidos por mayoría absoluta, y sujetos a rendición de cuentas, alternabilidad y revocatoria del mandato.

Dentro del análisis situacional del sector se identificaron algunos problemas en el Recinto Estrella Grande del Cantón Mocache, como son: débil gestión de la organización del territorio, bajo rendimiento de la actividad productiva, bajo nivel de escolaridad, la población no goza de buena salud y pésimos servicios básicos. Adicionalmente tienen problemas en la aplicación de tecnologías apropiadas que afectan el rendimiento en sus cultivos, el uso de semillas no calificadas, prácticas inadecuadas en el tratamiento de residuos orgánicos, y el uso incorrecto de agroquímicos, siendo estos los factores que más inciden en la disminución de sus ingresos.

\section{Actividades agrícolas en la zona}

Se estima que el área cultivada en este sector llega a 414,81 hectáreas de las cuales el $82,40 \%$ está destinada al cultivo de maíz, el 7,22\% al cultivo de arroz, el 4,27\% al de cacao (CCN51), el 5,76\% al cultivo de gandul y el 0,35\% al cultivo de otras variedades como maracuyá, papaya y tomate (GADP Los Ríos, 2015).

El 100\% de las familias del territorio siembran maíz, el 40\% además siembra arroz, cacao y gandul, y en menor escala maracuyá, papaya y tomate. En cuanto a la producción animal sólo se limita a la crianza de gallinas, patos y crianza de cerdos, esta actividad es para alimentación familiar.

Uno de los principales resultados que se esperaba con este proyecto es la de establecer sistemas agroforestales y de riego que aseguren la provisión de agua e iniciar la implementación de un plan de reforestación para la protección de fuentes de agua en al menos 30 ha de esteros y pajones.

\section{Evaluación de resultados e impactos}

Para evaluar el impacto socio-económico del proyecto de riego participaron: el equipo técnico del departamento de riego y drenaje de la Prefectura de los Ríos, comités de Gestión, de Veeduría y los estudiantes del octavo semestre de la Carrera de Economía de la Universidad Técnica Estatal de Quevedo-UTEQ, quienes reciben como asignatura la planificación económica y social.

Con la aplicación del proyecto se llegó a:

1.- Establecer 76 hectáreas con sistemas agroforestales (cacao, plátano, soya, aguacate, limón y guabo) con sistemas de riego que aseguran la provisión de agua.

2.- Implementar un plan de reforestación para la protección de áreas de interés para la conservación de los recursos naturales y protección de fuentes de agua en al menos 30 ha de esteros y pajones.

Lo que se pretende en este trabajo investigativo es evaluar los resultados y el impacto socio-económico, así como los ambientales que ha generado la puesta en marcha del proyecto de desarrollo rural "Mejoramiento de la producción agrícola a través de la implementación de sistemas agroforestales y riego parcelario, Cantón Mocache-Provincia de Los Ríos". Se trata de valuar las acciones realizadas (actividades cumplidas) y los impactos que estos han generado en las familias involucradas en el proyecto. De la misma forma se verificaría los cambios que en cada familia y en general en el territorio se han producido a finalizar el proyecto. En resumidas cuentas, cerciorar que la implementación de este proyecto ha contribuido al mejoramiento de la calidad de vida de 60 familias del territorio Estrella Grande (recinto del sector sur cantón Mocache). La implementación de un sistema de riego permite incre-

El sistema de riego parcelario como herramienta para el desarrollo de comunidades rurales. Evaluación del caso cantón Mocache- provincia de Los Ríos-Ecuador 
mentar la productividad del sector, la generación y obtención de nuevas oportunidades de trabajo, contribuyendo al mejoramiento del nivel de vida de los productores beneficiados además del desarrollo de los territorios (Bermúdez et al, 2010).

Así mismo, para que tenga éxito el desarrollo de los territorios bajo un sistema de riego, es imperioso considerar a la organización de regantes, los encargados de planificar, impulsar y ejecutar las estrategias del desarrollo en el territorio de riego. Para eso se debe fortalecer y desarrollar los procesos colectivos dentro de la organización de regantes. Estas organizaciones no sólo deben preocuparse por la gestión de la administración, operación y mantenimiento del sistema de riego, sino contribuir con asistencia técnica, proveer de insumos agrícolas, conseguir nuevos mercados para sus productos, gestiones ante instituciones públicas y privadas (Sosa y Larrea, 2014). Las decisiones para el desarrollo del territorio, deben ser compartidas y participativas entre todos los regantes, sustentadas en los principios de la economía social como: equidad, democracia, sustentabilidad y trabajo en equipo.

\section{Metodología}

La investigación se realizó en el Recinto Estrella Grande del sector sur del Cantón Mocache, Provincia de Los Ríos, ubicado en el centro norte de la provincia de Los Ríos, limita al norte con el Cantón Quevedo, al sur con los cantones Palenque, Vinces y Ventanas, al este con los cantones Quevedo y Ventanas y al oeste con los cantones Palenque en provincia de Los Ríos y el Empalme y Balzar en la provincia del Guayas. Tiene una superficie de $567,96 \mathrm{~km} 2$ que representa el $8 \%$ de la superficie provincial y su población proyectada por el INEC para el 2018 es de 42.720 habitantes (Ecuador en cifras, s.f.), de los cuales son hombres $52 \%$ y mujeres $48 \%$, el $79 \%$ de la población viven en el sector rural.

Las fuentes de información primaria las integraron los productores del sector, pertenecientes a las 60 familias beneficiarias del proyecto, quienes a su vez son usuarios del proyecto de riego, y los funcionarios del departamento de riego y drenaje del GADP de Los Ríos, con sede en la ciudad de Puebloviejo. La información secundaria se obtuvo de los archivos de la asociación de agricultores del sector, así como el proyecto implementado. La indagación básica se recolectó mediante un formulario, aplicado a los usuarios del servicio de riego.

La población objeto de esta investigación las conformaron los usuarios, constituida por productores agrícolas pertenecientes a las 60 familias del sector beneficiarias del proyecto de riego. Para obtener los datos informativos se realizó lo siguiente:

- Encuesta a usuarios, durante los meses de julio y agosto de 2018

- Entrevistas individuales a los funcionarios del departamento de riego y drenaje del GADP

- Observación directa

Para la consolidación de los resultados se tabuló la información y luego se procedió a hacer uso de técnicas de la estadística descriptiva.

\section{Resultados}

El universo fue de 60 agricultores, se pretendió realizar la encuesta a todos, pero no estuvieron presente 20 de ellos porque estaban cumpliendo con sus actividades agrícolas y familiares. Las encuestas fueron, por tanto, contestadas por 40 agricultores pertenecientes a la Asociación de trabajadores Agrícolas "Estrella Grande", que son el 67\% del universo, siendo una muestra bastante representativa, por cuanto significa más de los dos tercios. Luego de realizar la tabulación y el diagnóstico con las encuestas desarrolladas se presentan los siguientes resultados: De los encuestados, el $70 \%$ son de sexo masculino. La edad se encuentra entre los 30 y 70 años, lo cual nos da a entender la experiencia en el área de producción agrícola de las personas del sector.

Los datos de escolaridad, nos muestra que la mayoría de los encuestados, el 65\% tienen una formación primaria, mientras que un 25\% alcanzó estudios secundarios, cabe anotar que entre los participantes no se encontraron personas con educación universitaria.
En cuanto al tema que nos ocupa, como es el sistema de riego implementado en el recinto Estrella Grande del cantón Mocache, debemos indicar que en 2015 , el $90 \%$ de la población de este sector se abastecía del líquido vital por medio de pozos y un 10\% lo hacía de manera superficial, ya sea por embalses, pantanos u otras fuentes no subterráneas.

Los beneficiarios mantienen un rango de entre 1 a 5 hectáreas. La mayoría de ellos (58\%) poseen una hectárea, en la cual utilizan el sistema de riego, un $20 \%$ posee dos hectáreas, mientras que un $10 \%$ poseen hasta cinco hectáreas.

Así mismo, podemos observar que el 100\% utiliza el método de aspersión para el riego de sus tierras, utilizando motobombas para su sistema de riego. La frecuencia de utilización del sistema de riego de los diferentes productores del sector lo hace un $77 \%$ en sus predios de manera semanal, mientras que un $23 \%$ del total de los encuestados lo hacen mensualmente.

De la población encuestada, el $72 \%$ reconoce que se han realizado visitas a la zona del proyecto por parte de los técnicos para verificar el sistema, mientras que el restante $28 \%$ manifiesta que no ha tenido ese seguimiento por parte de los técnicos.

El estudio reveló que de los beneficiarios que han realizado talleres sobre la situación actual del funcionamiento del sistema de riego implementado, un $42,5 \%$ se ha capacitado en análisis y estimación de las eficiencias de captación, conducción y distribución además, el 50\% manifestó que se han recibido talleres de formas de organización existentes y con $37,5 \%$ respectivamente se han capacitado en cuanto a la conformidad de la directiva y sistemas de cargos y por último con 33\% se han capacitado en gestiones internas: formas de elección, responsabilidades por cargos para la operación y mantenimiento, sanciones, mecanismos de resolución de conflictos y aportes (Tabla 1).

Tabla 1. Realización de talleres comunitarios para recoger información de los usuarios respecto a los siguientes puntos:

\begin{tabular}{|c|c|c|c|c|c|}
\hline & $\mathrm{Si}$ & $\%$ & No & $\%$ & Total \\
\hline $\begin{array}{l}\text { - Estado actual de funcionamiento del sistema de riego } \\
\text { implementado }\end{array}$ & 25 & 62,50 & 16 & 37,50 & $100 \%$ \\
\hline $\begin{array}{l}\text { - Análisis y estimación de las eficiencias de captación, } \\
\text { conducción y distribución. }\end{array}$ & 17 & 42,50 & 23 & 57,50 & $100 \%$ \\
\hline $\begin{array}{l}\text { - Formas de Organización existentes (asociación, comité, } \\
\text { comunidad de base, sindicato, otro) }\end{array}$ & 20 & 50,00 & 20 & 50,00 & $100 \%$ \\
\hline $\begin{array}{l}\text { - Conformidad de la directiva; sistema de cargos para } \\
\text { riego. } \\
\text { - Gestión Interna: formas de elección, responsabilidades }\end{array}$ & 15 & 37,50 & 25 & 62,50 & $100 \%$ \\
\hline $\begin{array}{l}\text { por cargos para la operación y mantenimiento, } \\
\text { sanciones, mecanismos de resolución de conflictos y } \\
\text { aportes. }\end{array}$ & 13 & 32,50 & 23 & 67,50 & $100 \%$ \\
\hline
\end{tabular}

Fuente: Elaboración propia a partir de la encuesta a agricultores de la zona (2018)

La situación que se pudo observar en el diagnóstico fue que el $65 \%$ del total de los encuestados, declaró que el agua es suficiente en cuanto a lo que se usa para el sistema de riego, y el 35\% que no es suficiente el agua para el riego en los predios.

En cuanto a los principales problemas declarados entorno al sistema de riego, el $48 \%$ dijo que el principal problema es la falta de agua en los caudales, un 40\% manifestó que existe desorganización por parte de los regadores, un 35\% dice que por la falta de canales para conducción de agua y un $25 \%$ respectivamente manifiestan que se dan por la acequia malas condiciones, falta de mantenimiento, malas captaciones de agua y filtraciones (Tabla 2). 
Tabla 2. Principales problemas declarados en torno al sistema de riego

\begin{tabular}{|c|c|c|}
\hline Problema & Cantidad & $\%$ \\
\hline - Falta de Agua, caudal & 19 & 47,50 \\
\hline $\begin{array}{l}\text { - Falta de canales para la } \\
\text { conducción del agua }\end{array}$ & 14 & 35,00 \\
\hline $\begin{array}{l}\text { - Acequia en malas condiciones, } \\
\text { falta de mantenimiento, } \\
\text { tapada de basura, etc. }\end{array}$ & 10 & 25,00 \\
\hline $\begin{array}{l}\text { - Desorganizados los regadores, } \\
\text { turnos de pocas horas }\end{array}$ & 16 & 40,00 \\
\hline
\end{tabular}

Fuente: Elaboración propia a partir de la encuesta a agricultores de la zona (2018)

Por voluntad e iniciativa de los productores agrícola del Recinto Estrella Grande del Cantón Mocache, manifiestan que están dispuestos a mejorar su sistema de riego, el 100\% de la población encuestada manifestó que sería muy beneficioso para mejorar la producción y el sector.

En cuanto a las capacitaciones por parte del GADP, los productores manifestaron con un $93 \%$ que, si la han recibido, mientras que un $7 \%$ manifestó no haber recibidos dichas capacitaciones. Los medios que buscan los productores para asesorarse técnicamente, en cuanto alguna situación que se les presenta en su propiedad por el sistema de riego, el $37 \%$ recurren a ingenieros agrónomos, mientras que en un 30\% lo hacen en base a sus conocimientos propios y experiencia adquirida, en un $20 \%$ recurren a lugares de venta de insumos agrícolas y en un $8 \%$ y $5 \%$ respectivamente solicitan a particulares y vecinos o conocidos.

El 100\% de los beneficiarios poseen derecho de uso de agua respectivamente para sus sistemas de riegos implementados en los diferentes predios. Mientras que el $58 \%$ de la población encuestada mantiene su canal de abastecimiento del sistema de riego de 0 a 100 metros, mientras que el $43 \%$ lo ubica de 100 a 500 metros de distancia. En cuanto al grado de eficiencia del sistema de riego implementado, el $31 \%$ de la población manifiesta estar muy satisfecha, el $62 \%$ se encuentra satisfecha, mientras que un $7 \%$ de la población dijeron estar insatisfecha (Tabla 3).

Tabla 3. Con respecto al riego de su parcela, se encuentra usted

\begin{tabular}{lcc}
\hline Respuestas & Cantidad & $\%$ \\
\hline Muy satisfecho & 12 & 31 \\
Satisfecho & 24 & 62 \\
Insatisfecho & 3 & 7 \\
Total & 39 & 100
\end{tabular}

Fuente: Elaboración propia a partir de la encuesta a agricultores de la zona (2018)

Para el $45 \%$ de los productores agrícolas su producción es regularmente diversa, en un 33\% manifestaron ser muy diversa y el $22 \%$ del total de población respondió tener una producción poco diversa.

Con la implantación del sistema de riego parcelario en el recinto, el $65 \%$ ha desarrollado nuevos sistemas agroforestales (cacao, plátano, soya, aguacate, limón, guabo), el 52,5 \% en la ampliación de áreas de cultivo, mientras que el 40\% manifestó que sí se implementó un plan de reforestación para protección de áreas de interés para la conservación de recursos naturales. Entre un $10 \%$ y $27,5 \%$ ha conseguido un mejoramiento de la tecnología y el $27,5 \%$ ha incrementado la demanda de los trabajadores en sus parcelas (Tabla 4).

Tabla 4. Principales efectos generados por la implementación del riego parcelario en el recinto Estrella Grande del sector sur del cantón Mocache

\begin{tabular}{rlcc}
\hline Tipos & & Cantidad & $\%$ \\
\hline - & $\begin{array}{l}\text { Establecimiento de nuevos sistemas agroforestales } \\
\text { (cacao, plátano, soya, aguacate, limón, guabo) }\end{array}$ & 26 & 65,00 \\
- & $\begin{array}{l}\text { Implementación de un plan de reforestación para la } \\
\text { protección de áreas de interés para la conservación de }\end{array}$ & 16 & 40,00 \\
$\quad \begin{array}{l}\text { recursos naturales. } \\
\text { - Ampliación de áreas de cultivo }\end{array}$ & 21 & 52,50 \\
- Incremento de la demanda de trabajo & 11 & 27,50 \\
\hline
\end{tabular}

Fuente: Elaboración propia a partir de la encuesta a agricultores de la zona (2018)
Acerca de la percepción de los productores, sobre el mejoramiento del nivel de vida por la implementación del proyecto en el sector, un 95\% del total de los encuestados manifestaron que si se percibe el mejoramiento del nivel de vida, por cuanto se ha diversificado la producción agrícola en sus cultivos través de la implementación de sistemas agroforestales y de acciones mancomunadas para fortalecer la organización (Tabla 5).

\begin{tabular}{lcc} 
Tabla 5. Percepción de mejoramiento del nivel de vida por el establecimiento del proyecto. \\
\hline Respuestas & Cantidad & $\%$ \\
\hline Se percibe mejoramiento del nivel de vida & 38 & 95 \\
& 1 & 2,5 \\
No se percibe mejoramiento del nivel de vida & 1 & 2,5 \\
Ns/Nc & 40 & 100 \\
Total & & \\
\hline
\end{tabular}

Fuente: Elaboración propia a partir de la encuesta a agricultores de la zona (2018)

Para concluir, podemos observar que del 100\% de la población identificó las deficiencias técnico económicas que hay dentro del sistema de riego implementado, para el 35\% existe una presión deficiente del riego, el 30\% manifestó que el proyecto es muy costoso, el $25 \%$ dijo que la infraestructura es defectuosa y en un rango del $12,5 \%$ y $20 \%$ respectivamente, identificaron la baja capacidad del proyecto y la administración ineficiente del mismo.

\section{Discusión}

Es evidente la importancia de implementar sistemas de riego parcelario en el sector agrícola, la incorporación de este sistema cambia considerablemente la vida de los pequeños agricultores en el campo. El equipo técnico del GADP de Los Ríos debería otorgar capacitación y asistencia técnica a los pequeños agricultores para una buena gestión integral del riego. Es necesario que los regantes se organicen de la mejor forma para obtener resultados esperados.

Se recomienda realizar jornadas de sensibilización y motivación entre los asociados con frecuencia, para esto se pueden aprovechar las sesiones de capacitaciones. Una de las causas de fracasos entre las organizaciones asociativas de campesinos es que con el tiempo los miembros van perdiendo la motivación y disminuyen el esfuerzo. Es necesario concienciar a los asociados del esfuerzo que deben realizar y mantenerlos enfocados hacia una meta común para que ese esfuerzo no disminuya y se puedan alcanzar los objetivos de la asociación.

\section{Conclusiones}

El sistema de riego implementado por el GADP de Los Ríos sí ha beneficiado a los agricultores en el sector Estrella Grande, porque les ha permitido desarrollar en sus propiedades sistemas agroforestales (cacao, plátano, soya, aguacate, limón y guabo). Su producción agrícola ha mejorado en relación a años anteriores y su nivel de vida está en mejores condiciones.

Está pendiente la conclusión del plan de reforestación para la protección de áreas de interés para la conservación de los recursos naturales y protección de fuentes de agua, en al menos 30 hectáreas de esteros y pajones, pocos agricultores han desarrollado esta actividad. Se han incrementado sus ingresos, por la venta de sus productos agrícolas. En definitiva los agricultores están satisfechos por la eficiencia del sistema de riego implementado.

Se presenta una estructura de organización sólida con participación de todos sus miembros. Sin embargo, la organización muestra una limitada capacidad técnica y administrativa. Es necesario impulsar la autoformación en la asociación, aprovechando y rentabilizando los propios recursos y experiencias, de las capacidades de sus miembros. Creando redes de intercambio de formación entre asociaciones, con los recursos formativos de cada una. Compartir aquellos conocimientos y habilidades en los que cada asociación es más fuerte. De la misma forma promover convenios de cooperación con instituciones del Estado para fortalecer su capacidad productiva. 
La evaluación del cumplimiento del proyecto agroforestal y de riego parcelario, permite concluir que los resultados han sido mayoritariamente positivos y que el propósito de mejorar las condiciones de vida y de trabajo de los agricultores, se ha concretado.

Estos proyectos no deberían hacerlos únicamente los GADS sino también las Instituciones de Educación Superior por medio de charlas y asesoramientos. La Universidad Técnica Estatal de Quevedo, como parte de su función de contribuir con la sociedad, correspondería ser la precursora en promover el concepto de economía popular y solidaria, llegando a estas asociaciones a través de los proyectos de vinculación con la comunidad que normalmente se realizan.

El saber llegar a las comunidades de los campesinos, conociendo su idiosincrasia, es fundamental para poder motivarlos y convencerlos, que un trabajo en conjunto, en función de objetivos comunes, será lo que les permitirá tener una mayor productividad y a la vez mayores ingresos económicos.

Los proyectos que en adelante se realicen, deben considerar la problemática del sector para que tengan éxito. Se concluye que el elaborado y puesto en marcha por la Prefectura de la Provincia de Los Ríos, ha dado un resultado positivo en vista de que la amplia mayoría de los usuarios han visto el mejoramiento de su calidad de vida. En este caso, se detectó que el riego es un problema crónico, a pesar de tener fuentes hídricas relativamente cercanas, no las han podido aprovechar. La asistencia técnica e inversión para solucionar esta problemática por parte del GADP Los Ríos, ha sido una decisión acertada por parte de esta entidad.

Los niveles de satisfacción por el haber implementado este sistema de riego es alto, lo que corrobora que ha existido un estudio previo para dar solución a una necesidad del sector agrario de esta zona de la provincia, cuya economía se basa en la producción agrícola.

\section{Referencias}

Baca, N. y Herrera, F. 2016. Proyectos sociales. Notas sobre su diseño y gestión en territorios rurales. Convergencia. Revista de Ciencias Sociales, vol. 23, núm. 72, septiembre-diciembre, 2016, pp. 69-87, Universidad Autónoma del Estado de México.

Bermúdez, L., Rodríguez, L., y Páez, A. 2010. Impactos socioeconómicos y ambientales del Proyecto de Riego y Drenaje del Valle del Alto Chicamocha y Firavitoba. Boyacá (Colombia), Agron. colomb., Volumen 28, Número 2. p. 337 344, 2010. Impreso 0120-9965.

Constitución de la República del Ecuador Asamblea Constituyente 2008. Registro oficial 449. Art. 263. Ecuador.

Ecuador en cifras (s.f.). Proyección de la población ecuatoriana, por años calendario, según cantones http://www.ecuadorencifras.gob.ec/documentos/ webinec/Poblacion_y_Demografia/Proyecciones_Poblacionales/proyeccion_ cantonal_total_2010-2020.xlsx Recuperado el 08/03/2019

FAO, 2004: "Política de desarrollo agrícola Conceptos y principios". Organización de las Naciones Unidas para la Agricultura y la Alimentación, Roma, 200.

GADP Los Ríos. 2016. Suplemento Rendición de Cuentas, Riego y Drenaje. Gobierno Autónomo Descentralizado Provincial de Los Ríos, www.agricultura. gob.ec/el-plan-nacional-de-riego/. Recuperado el 27/05/2018.

Juárez Sánchez-Rubio, C. 2008. Avance y repercusión del regadío sostenible en las zonas rurales de Alicante: reto y oportunidades. Departamento de Análisis Geográfico Regional y Geografía Física. Universidad de Alicante Investigaciones geográficas, $n^{\circ}$ 45. pp. 29-61.

Ley Orgánica de Economía Popular y Solidaria y del sector Financiero Populary Solidario. 2011. Registro Oficial (Separata), 2011-05-10, núm. 444, 59 págs.

Lozano, I. y Restrepo, J. 2016. El papel de la infraestructura rural en el desarrollo agrícola en Colombia, Coyuntura Económica. Volumen XLVI, No. 1, Junio de 2016, pp. 107-147. Fedesarrollo, Bogotá - Colombia.

MAGAP. 2102. Plan nacional de Riego y Drenaje 2012-2026. Ministerio de
Agricultura, Ganadería, Acuacultura y Pesca, Acuerdo No. 342. Ediciones Legales 2016. Ecuador

Martínez de Anguita d' Huart, P. 200). Desarrollo rural sostenible. McGrawHill Interamericana de España S.L., $1^{a}$ ed.

Sosa, B. y Larrea, D. 2014. La tecnificación de la agricultura familiar bajo riego en Ecuador. El riego, planificación y tecnificación, Foro de los Recursos Hídricos. CESA. Quito-Ecuador, pp. 120-122. 